\title{
Photophysical Consequences of Coupling Bacteriochlorophyll a with Serine and its Resulting Solubility in Water"
}

\author{
I. Eichwurzel ${ }^{\star 1}$, H. Stiel ${ }^{1}$, K. Teuchner ${ }^{1}$, D. Leupold ${ }^{1}$, H. Scheer ${ }^{2}$, Y. Salomon ${ }^{3}$ and A. Scherz ${ }^{3}$ \\ ${ }^{1}$ Max-Born-Institut fuer Nichtlineare Optik und Kurzzeitspektroskopie, Berlin, Germany; \\ ${ }^{2}$ Botanisches Institut der Universität München, München, Germany and \\ ${ }^{3}$ Department of Plant Science, The Weizmann Institute of Science, Rehovot, Israel
}

Received 10 January 2000; accepted 3 May 2000

\begin{abstract}
We investigated the dependence on solvents of optical absorption and emission of the bacteriochlorophyll $a$-serine (BChl-ser), a water soluble bacteriochlorophyll (BChl) derivative. Comparison between the experimental data and those collected for $\mathrm{BChl}$ in nonaqueous solvents shows that only a minor interaction takes place between serine and the macrocycle's $\pi$-electron system. Nevertheless, the coupling with serine results in a small enhancement of the nonradiative relaxation rate from the first excited singlet state $S_{1}$. In buffered aqueous solution ( $\mathrm{pH}$ $=7.4$ ), the Stokes shift of the BChl-ser fluorescence and its nonradiative relaxation rate are enhanced compared with those in nonaqueous solutions (Scherz, A., S. Katz, Y. Vakrat, V. Brumfeld, E. Gabelmann, D. Leupold, J. R. Norris, H. Scheer and Y. Salomon (1998) Photosynthesis: Mechanisms and Effects, Vol. V (Edited by G. Garab), pp. 4207-4212. Kluwer Academic, Dordrecht.), probably as a result of a hydrogen bonding between the BChl macrocycle and the water molecules. In aprotic solvents, without hydrogen bonds, the permanent dipole moment of the first excited singlet state in both BChl and BChl-ser is increased compared with the ground state by at least 2.5 Debye.
\end{abstract}

\section{INTRODUCTION}

The photophysics of bacteriochlorophyll $a(\mathrm{BChl}) \dagger$ has received considerable attention during the last three and a half decades to understand the functions of the molecule as a component in antennae and in reaction centers of photosynthetic bacteria. $\mathrm{BChl}$ is responsible for the intense absorption

IIPosted on the web on 24 May 2000.

*To whom correspondence should be addressed at: Max-Born-Institut fuer Nichtlineare Optik und Kurzzeitspektroskopie, MaxBorn Str. 2a, D-12489 Berlin, Germany; e-mail: eichwurzel@ mbi-berlin.de

$\dagger$ Abbreviations: BChl, bacteriochlorophyll $a$; BChl-ser, $17^{3}$ ester between bacteriochlorophyllide $a$ and serine methyl ester; BPheideser, bacteriopheophorbide-serine; DMSO, dimethyl sulfoxide; IC, internal conversion; ISC, intersystem crossing; OD; optical density; PBS, phosphate-buffered solution; PDT, photodynamic therapy; THF, tetrahydrofuran.

(C) 2000 American Society for Photobiology 0031-8655/00 \$5.00+0.00 and the very fast energy transfer through the antenna system and eventually to the reaction center, as well as for the following ultrafast charge separation across the photosynthetic membrane.

In addition to the ongoing research of $\mathrm{BChl}$ in the context of photosynthesis, there is a growing interest in the medical applications of $\mathrm{BChl}$ and its derivatives, in particular, in considering the spectroscopic and photochemical properties of $\mathrm{BChl}$, which make it a promising sensitizer for photodynamic therapy (PDT) $(1,2)$.

However, the poor solubility of the native compound in water limits its application to micellar or liposomic formulation. Furthermore, the hydrophobicity of the sensitizer may also slow down its clearance from normal tissue $(3,4)$.

Recently it became possible to render BChl water-soluble by esterification with a polar amino acid such as serine methyl ester (5). Initial tests $(3,4)$ have indeed shown that a $17^{3}$ ester between bacteriochlorophyllide $a$ and serine methyl ester (BChl-ser) is an improved PDT sensitizer compared with BChl, which calls for basic photophysical investigations of this new molecule.

$\mathrm{BChl}$ is known to show a different solvatochromism in polar and nonpolar solvents (6). Here we focused on a series of polar solvents, including water, and investigated the dependence of the $Q_{x}$ and $Q_{y}$ transition energies, the fluorescence lifetime and the fluorescence quantum yield of BChlser on the solvent tested. In interpretating the data we aimed to separate the influences of: (1) the serine interactions with the BChl moiety; and (2) the effect of an aqueous environment on the photophysical behavior of BChl. We show that the spectroscopic properties of the BChl moiety are practically not affected by the serine residue. The evaluation of this data allows, for the first time, to investigate the photophysical properties of monomeric $\mathrm{BChl}$ in an aqueous environment.

\section{MATERIALS AND METHODS}

Materials. BChl-ser was synthesized as described in Fiedor et al. (5). All solvents used were of spectroscopic grade and were acquired from the following sources. Diethylether, dichloromethane, acetone, dimethylsulfoxide (DMSO), ethanol, chloroform, methanol, tetrahydrofuran (THF) and dioxane from Merck Uvasol; isobutanol, acetophenone and pyridine from Aldrich; quinoline (distilled before measuring) from Chemapol; phosphate-buffered solution (PBS, pH $=7.4$ ) from Sigma. 


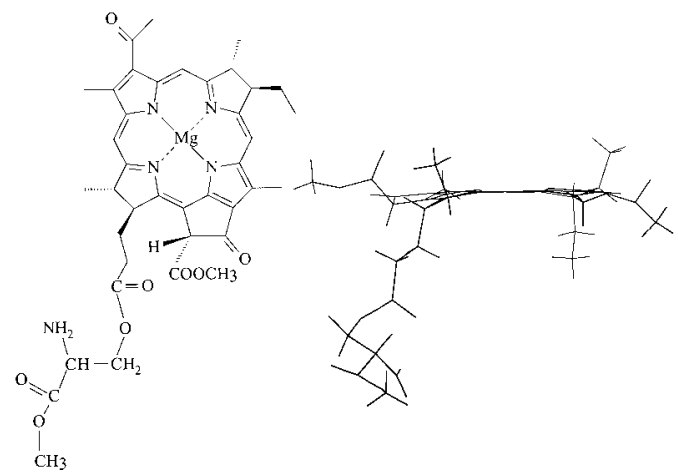

Figure 1. Structural formula of BChl-ser (left) and conformation (side view, right) calculated with a molecular mechanics force field (30).

Experimental. Absorption spectra were recorded using a Lambda19 spectrometer from Perkin-Elmer.

A Fluorolog model FL-112 (Spex Industries) fluorimeter was used to measure the steady-state fluorescence. To avoid reabsorption effects, all fluorescence data were taken from solutions with low optical densities (OD $<0.1$ at the absorption maximum). BChl in diethyl ether $\left(\Phi_{f l}=0.19\right)$ was used as a standard to calculate the absolute values of the fluorescence quantum yields $\Phi_{f t}$ in other solvents and of other pigments (7). The fluorescence emission was excited at $740 \mathrm{~nm}$.

Fluorescence lifetimes were measured using a nitrogen laser (MSG 850/LTB Berlin)-pumped dye laser (pulse duration 400 ps, excitation wavelength $770 \mathrm{~nm}$ ) as an excitation source and a boxcarintegrator detection system (8).

\section{RESULTS AND DISCUSSION}

\section{Solvent effects on the absorption}

The photophysical properties of BChl-ser (see Fig. 1) are mainly determined by the macrocycle's $\pi$-electron system and the coordinated central $\mathrm{Mg}$ atom. This part of the molecule has a basically planar structure.

At first, the absorption behavior of BChl-ser will be compared with that of BChl. Although BChl is insoluble in water it can be dissolved in several organic solvents (e.g. [6]). The UV-visible-near-IR absorption spectrum of BChl in acetone is shown in Fig. 2. It consists of four main bands termed $Q_{y}$, $Q_{x}, B_{x}, B_{y}$ in the order of increasing energy. The last two comprise the Soret band. The first two result from transitions to the first and second excited singlet states, respectively $(9,10)$. The absorption spectra of BChl-ser and BChl in the same polar solvent are similar (cp Fig. 2).

Limantara et al. (6) investigated the optical absorption of $\mathrm{BChl}$ in numerous solvents. In accordance with the theory of Nagae et al. ([11], cp also [12]), they observed a linear dependence of the $Q_{y}$ and $Q_{x}$ absorption energies on the parameter $R(n)=\left(n^{2}-1\right) /\left(n^{2}+2\right)$, determined by the refractive index $n$. Different slopes were obtained in polar and nonpolar solvents. The $Q_{y}$ transition energies of BChl (taken from Limantara et al. [6]) and of BChl-ser (this work) are compared for polar solvents in Fig. 3. Apparently, the $S_{0} \rightarrow$ $S_{1}$ transition energy of BChl-ser and BChl follows the same dependence on $R(n)$. Note that $R(n)$ of BChl-ser in aqueous solution (PBS, $c p$ below) fits well into the general $R(n)$ dependence. The linear regression in Fig. 3 is faithfully represented by

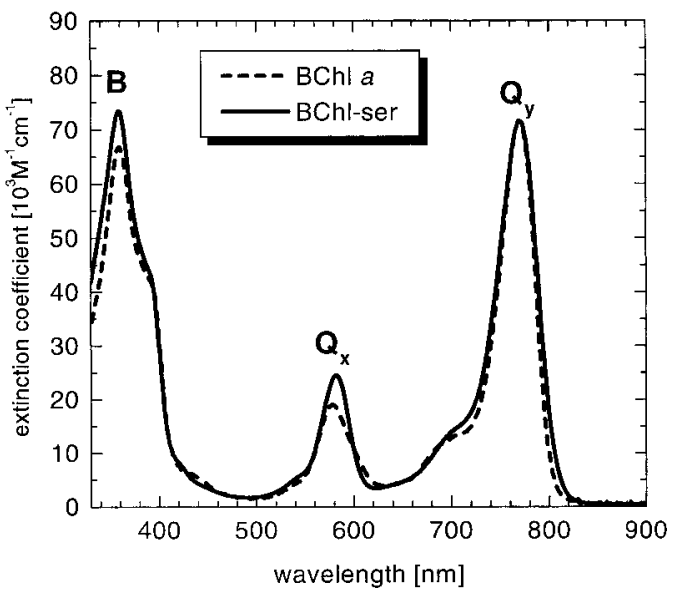

Figure 2. Absorption spectra of $\mathrm{BChl}$ and $\mathrm{BChl}-$ ser dissolved in acetone.

$$
\nu Q_{y}\left[\mathrm{~cm}^{-1}\right]=1895 \cdot R(n)+13380, \sigma=47.5,
$$

where $v$ is the wavenumber of the $Q_{y}$ absorption maximum. The coefficients correspond to the data given by Limantara et al. (6). The extrapolation to aqueous solvents indicates that the position of the $Q_{y}$-band can also be used to derive a local, isotropic $n$ for protein environments, as long as there are no pigment-pigment interactions or specific interactions with particular amino-acid residues.

The spectral position of the $Q_{x}$ absorption band of BChl depends strongly on the coordination state of the central metal atom. This is mainly because of electron density migration between the ligand and the metal that modifies the metal covalent radius and the electrostatic interaction of the metal with the electron density at the $\pi$-atoms in the frontier molecular orbitals (13). When penta-coordinated, the $Q_{x}$-band is around $570 \mathrm{~nm}$ and the $\mathrm{Mg}$ is localized outside the plane of the macrocycle. If the $\mathrm{Mg}$ is hexa-coordinated, it is positioned in the plane of the macrocycle (14) and the $Q_{x}$-band shifts to around $600 \mathrm{~nm}$. This assignment, which was originally based on the solvent dependence of the absorption

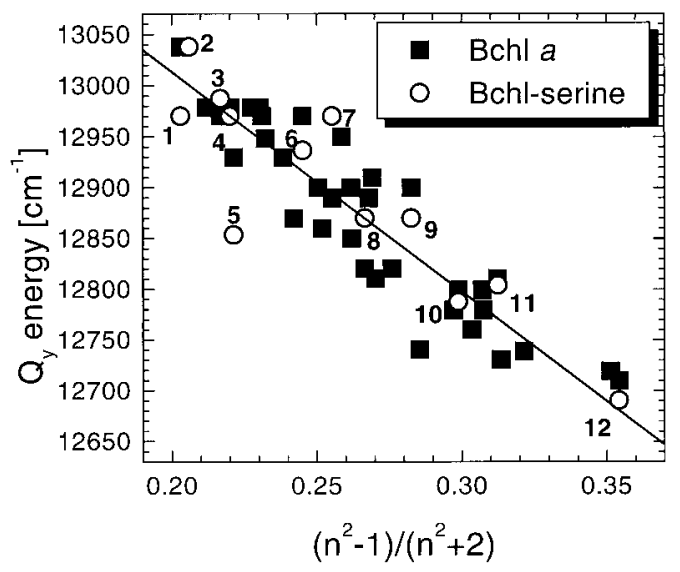

Figure 3. Absorption maxima of the $Q_{y}$ band of $\mathrm{BChl}$ (from Limantara et al. [6]) and BChl-ser. Only those solvents that were used in this study for BChl-ser are marked by numbers: 1, methanol; 2 , PBS; 3, diethyl ether; 4, acetone; 5, ethanol; 6, THF; 7, dichloromethane; 8, chloroform; 9, DMSO; 10, pyridine; 11, acetophenone; 12 , quinoline. 
Table 1. Coordination number $\mathrm{N}$ of the central $\mathrm{Mg}$ and spectral position of the $Q_{x}$ absorption band (in $\mathrm{nm}$, in parentheses) of BChl (from Limantara et al. [6]) and of BChl-ser

\begin{tabular}{lll}
\hline Solvent & BChl $a$ & BChl-ser \\
\hline Methanol & $6(607)$ & $6(605)$ \\
Diethyl ether & $5(573)$ & $5(580)$ \\
Acetone & $5(577)$ & $5(580)$ \\
Ethanol & $6(607)$ & $6(606)$ \\
DMSO & $6(608)$ & $6(607)$ \\
& $5(580)$ & $5(583)$ \\
Isobutanol & $6(604)$ & $6(599)$ \\
Chloroform & $5(580)$ & $5(581)$ \\
PBS (pH = 7.4) & - & $6(607)$ \\
\hline
\end{tabular}

(15), has been supported by Raman spectroscopy $(6,14,16)$ and extended X-ray absorption fine structure (13). Although there are further variations depending on the ligand strength (between 575 and $585 \mathrm{~nm}$ if the $\mathrm{Mg}$ is penta-coordinated and $593-610 \mathrm{~nm}$ if it is hexa-coordinated), it is possible to assign the coordination state by absorption spectroscopy.

Following this approach, the coordination numbers of the $\mathrm{Mg}$ atom in BChl and BChl-ser are identical in each of a variety of solvents (Table 1), indicating that the serine residue has no influence on the coordination of the central $\mathrm{Mg}$.

Taking this result together with the already described fact that the $Q_{y}$ transition energy of BChl-ser shows the same dependence on polar solvents as that of $\mathrm{BChl}$, we have concluded that there is a negligible influence of the serine bound to the $\mathrm{C}$-17-propionic acid side chain, on the $\pi$-electron system of the macrocycle. This allows us, for the first time, to obtain experimental information on the optical absorption and related molecular parameters of monomeric $\mathrm{BChl}$ in an aqueous environment by measuring the absorption properties of BChl-ser in aqueous solution. In this environment, the absorption band of the $Q_{x}$ transition is located at $607 \mathrm{~nm}$, suggesting that the $\mathrm{Mg}$ is hexa-coordinated in water and located in the plane of the macrocycle. The Mg interacts with two water molecules that are ligated along the $\mathrm{z}$-axis. Brereton and Sanders (17) showed by NMR spectroscopy that the hexa-coordinated $\mathrm{BChl}$ is more stable against oxidation than the penta-coordinated molecule (17). Nevertheless, it is noteworthy that BChl-ser in PBS is not (photo)chemically stable if the solution is equilibrated with air. Even exposure to the measuring light of the spectrophotometer was found degrading and Ar bubbling was required to inhibit (photo)chemistry.

The extinction coefficient, $\epsilon$, at the $Q_{y}$ maximum (in PBS) was determined to be $\epsilon=42000 \mathrm{M}^{-1} \mathrm{~cm}^{-1}$ by the following procedure: BChl-ser was dissolved in a defined volume of acetone and the absorption was recorded. After evaporation of the acetone under a stream of $\mathrm{Ar}$ and redissolving the BChl-ser in an identical volume of PBS, the optical density was recorded again, and $\epsilon$ was calculated from the absorbance ratio and the known $\epsilon$ in acetone. The extinction coefficient of BChl-ser dissolved in acetone, determined by atomic absorption spectroscopy (A. Scherz, unpublished), provided the same value $\left(71500 \mathrm{M}^{-1} \mathrm{~cm}^{-1}\right.$ at the $Q_{y}$ maximum) as BChl dissolved in acetone (18). The absorption of BChl-ser followed Lambert-Beer's law up to the maximum concentration used in the present study, $c=5 \times 10^{-5} \mathrm{M}$.

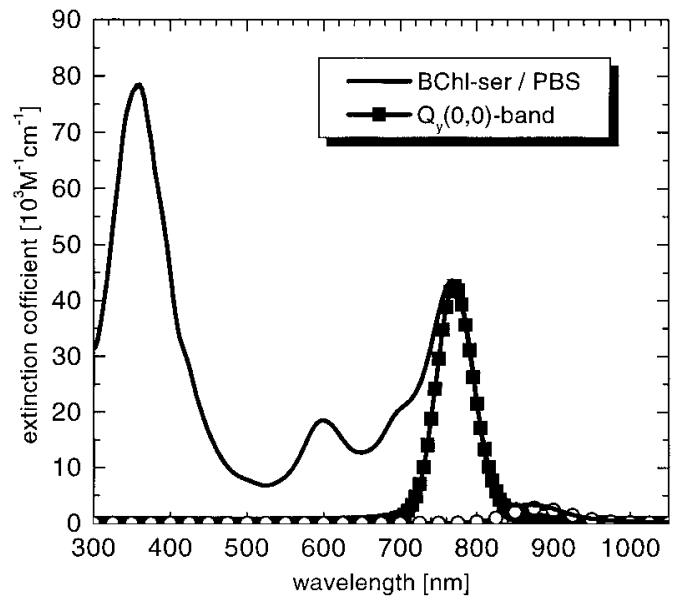

Figure 4. Absorption spectrum of BChl-ser in PBS, $\mathrm{pH}=7.4$ (solid line), and band analysis of the $0-0$ component of the $Q_{y}$-band by a Voigt-curve (squares, see text for details).

No concentration dependence could be observed; that means there is no indication of aggregated BChl-ser. These results are corroborated by recent studies of Katz (19).

As already shown in Fig. 2, BChl and BChl-ser differ somewhat in their absorption line-shapes. Therefore, we analyzed in more detail, the absorption line-shape of the $Q_{y}$ region of BChl-ser in PBS. The $0-0$ absorption band of the $S_{0} \rightarrow S_{1}$ transition was filtered out using a Voigt-profile $I_{\text {Voigt }}$. We started the analysis at $760 \mathrm{~nm}$ in order to minimize the influence of higher vibronic transitions on the $Q_{y}(0,0)$-band. The resulting band is shown in Fig. 4. Besides this band and the mentioned higher energy vibronic transitions, there is also a redshifted minor absorption band at $875 \mathrm{~nm}$ of yet unidentified origin.

The transition dipole moment $\mu$ of the $Q_{y}$ band (in Debye) has been determined by the following equation (16)

$$
\left|\mu_{i}\right|^{2}=\frac{9.18 \cdot 10^{-3} \cdot 9 n}{\left(n^{2}+2\right)^{2}} \int_{\nu_{1}}^{\nu_{2}} \frac{\epsilon_{i}}{v} d v
$$

where $n$ is the refractive index, $v$ the wavenumber and $\epsilon_{i}$ the molar extinction coefficient for transition $i$. In this equation, the Lorentz correction factor is used (20). Using this formula, we found that integration over the Voigt-type absorption band previously described, provides a transition dipole moment of $(5.2 \pm 0.5) \mathrm{D}$ for BChl-ser in PBS, $\mathrm{pH}=7.4$. This increases to $(6.6 \pm 0.5) \mathrm{D}$ if we consider the vibrational shoulder as well. The transition dipole moment of the entire $Q_{y}$ band of BChl-ser in acetone, determined in the same way, has a value of $\mu=(6.8 \pm 0.5) \mathrm{D}$. This value is comparable, within the experimental error, to that of BChl in acetone $(\mu$ $=6.5 \mathrm{D})$, which confirms the value determined in Scherz and Parson (16). Note that the dipole moment of the $S_{0} \rightarrow$ $S_{1}$ absorption transition of BChl-ser in PBS is comparable to that of the $S_{0} \rightarrow S_{1}$ absorption transition of BChl-ser in acetone, although the maximum extinction coefficient in PBS is significantly smaller, because of the absorption band broadening in water.

\section{Solvent effects on fluorescence}

In water, BChl-ser has an emission maximum at $\lambda_{e m}=801$ $\mathrm{nm}$, corresponding to a Stokes shift of $\nu_{S t}=540 \mathrm{~cm}^{-1}$. 


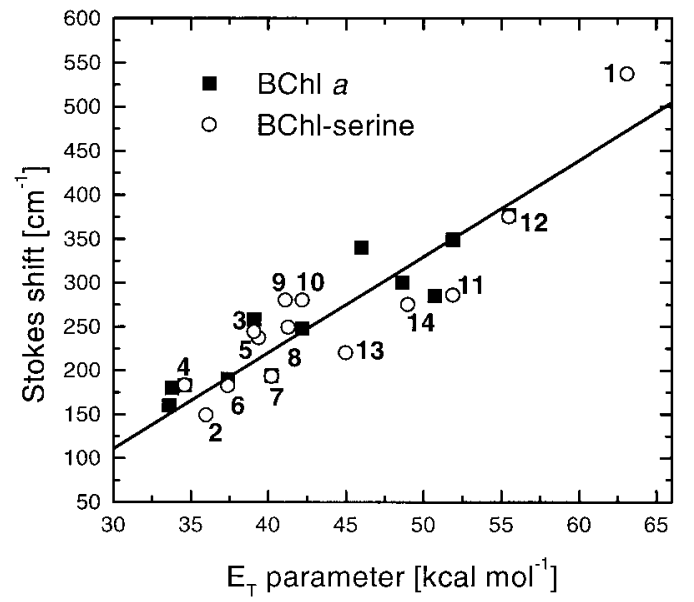

Figure 5. Dependence of the Stokes shift of BChl (from Connolly et al. [18]) and BChl-ser in different solvents on the Dimroth-Reichardt parameter $\mathrm{E}_{T}(21)$ of these solvents. Linear parameters of the fit for BChl-ser are $\Delta v_{\mathrm{St}}=10.7 \cdot \mathrm{E}_{T}-209$ (with a correlation coefficient of 0.91). The numbers give only those solvents in which BChl-ser was measured: 1, PBS; 2, diethyl ether; 3, THF; 4, quinoline; 5 , chloroform; 6 , pyridine; 7 , dichloromethane; 8 , acetophenone; 9 , acetone; 10 , isobutanol; 11 , ethanol; 12 , methanol; 13 , dioxane; 14 , DMSO.

The fluorescence properties of the molecule are determined by the permanent dipole moments of the ground and excited states, which in turn depend on the interaction between the fluorophore and the solvent molecules. These interactions result from the additive effect of electrostatic and electrodynamic forces, including dispersion, dipole-dipole interactions and hydrogen bonds. The Dimroth-Reichardt parameter $\mathrm{E}_{T}$ is a widely used global solvent polarity parameter (21) that accounts for this cumulative effect. The scatter plot in Fig. 5 shows the dependence of the Stokes shift $v_{S t}$ on the $\mathrm{E}_{T}$ values of the corresponding solvents.

For both BChl and BChl-ser, the Stokes shift has the same linear dependence on $\mathrm{E}_{T}$. BChl-ser dissolved in PBS has a Stokes shift that fits this linear dependence within the limits of uncertainty of the $\mathrm{E}_{T}$ parameter. Linear fits were also found for $v_{S t}$ as a function of $\alpha$ (hydrogen bonding donor ability), $\pi^{*}$ (ability to stabilize a charge distribution) and $\beta$ (hydrogen bonding acceptor ability) (22-24), with comparable quality. It is therefore likely that the fluorescence properties of BChl-ser in PBS are determined by the interaction between $\mathrm{BChl}$ and the solvent molecules and not by special effects, for example, the formation of aggregates, or a folding-back of the serine over the macrocycle, which even seems to be absent in vacuum following force-field calculations.

A frequently used expression to describe the effect of the solvent on the Stokes shift of fluorophores is the Lippert equation (12)

$$
\begin{aligned}
v_{S t} & =v_{a}-v_{f l} \\
& =\frac{2}{h c}\left(\frac{\epsilon-1}{2 \epsilon+1}-\frac{n^{2}-1}{2 n^{2}+1}\right) \frac{\left(\mu^{*}-\mu\right)^{2}}{a^{3}}+\text { const }
\end{aligned}
$$

where $h$ is Plancks constant, $c$ the speed of light, $a$ the radius of the cavity in which the fluorophore resides, $\mu^{*}$ the permanent dipole moment of the excited electronic state and $\mu$

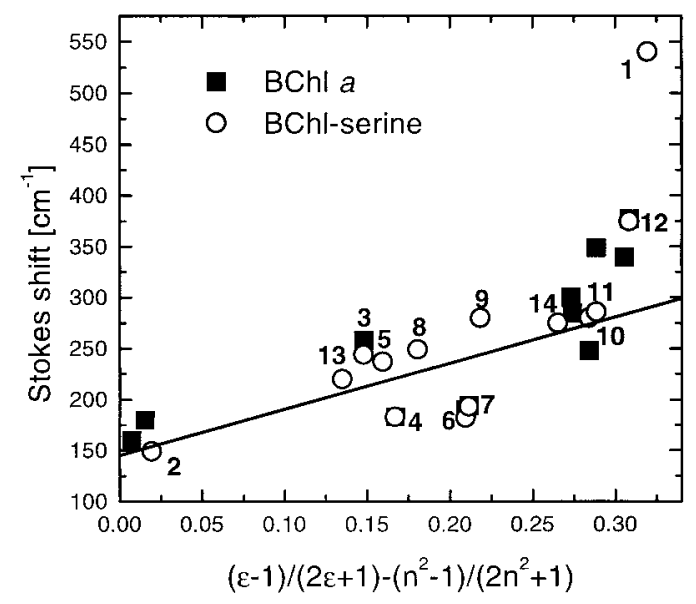

Figure 6. Stokes shift of BChl-ser and BChl as function of the refractive index $n$ and the dielectric constant $\epsilon$ (Lippert plot). The numbers give the solvents in which BChl-ser was investigated: 1 , PBS; 2, diethyl ether; 3 , THF; 4, quinoline; 5, chloroform; 6, pyridine; 7 , dichloromethane; 8 , acetophenone; 9 , acetone; 10 , isobutanol; 11, ethanol; 12, methanol; 13, dioxane; 14, DMSO.

that of the ground state. The values $v_{a}$ and $v_{f}$ represent the absorption and fluorescence maxima (in $\mathrm{cm}^{-1}$ ), respectively. The term contains the static dielectric constant $\epsilon,(\epsilon-1) /$ $(2 \epsilon+1)$, which reflects both the reorientation of the solvent dipoles and the redistribution of the electrons in the solvent molecules, whereas the term $\left(n^{2}-1\right) /\left(2 n^{2}+1\right)$, that contains the refractive index $n$, accounts only for the redistribution of electrons.

After excitation, a redistribution of electrons occurs in the excited electronic state, to which the surrounding solvent molecules respond by redistribution of their electrons. This process, termed solvent relaxation, influences the Stokes shift (12).

The Lippert plot for both BChl and BChl-ser can be fitted with the same linear parameters for most of the solvents (Fig. 6), indicating that serine introduces only a minor pertubation of the solvent shell around the BChl. The only exception is the value for BChl-ser dissolved in PBS, which lies significantly outside the fitted curve. However, the fit of $\mathrm{BChl}$ in methanol is already somewhat out of the linear curve. A likely reason for this discrepancy is the hydrogen bonding ability of water and, although less pronounced, methanol. For (Eq. 2) it is assumed that solvent molecules are treated as a continuum. Hence, specific solvent effects like hydrogen bonding are not considered, and with water the limits of the applicability of the Lippert equation are reached. The plot in Fig. 6 clearly shows the importance of the hydrogen bonds for the interaction between BChl-ser and water.

The similarity between the Stokes shifts of BChl and BChl-ser in the same solvents, combined with the absorption data, shows that the energy of the excited states of both molecules and the electronic density distribution in the frontier orbitals are the same. This means that the serine does not affect the spectral position of the $S_{0} \rightarrow S_{1}$ and $S_{1} \rightarrow S_{0}$ transitions.

An initial estimate of the change of the dipole moment from the ground state to the first excited state in solvents without hydrogen bonds can be computed using the Lippert 
Table 2. Fluorescence data of BChl-ser (this work) and BChl (from Connolly et al. [18], in parentheses)

\begin{tabular}{|c|c|c|c|c|c|c|}
\hline Solvent & $\lambda_{e m}(\mathrm{~nm})$ & $\Phi_{f l}$ & $\tau_{f l}(\mathrm{~ns})$ & $\tau_{0}[\mathrm{~ns}]^{*}$ & $v_{S t}\left(\mathrm{~cm}^{-1}\right)$ & $\begin{array}{l}k_{I C}+k_{I S C} \\
\left(\times 10^{8} \mathrm{~s}^{-1}\right)\end{array}$ \\
\hline PBS & 801 & 0.04 & 1.1 & 27.5 & 540 & 8.7 \\
\hline Methanol & 795 (793) & $0.11(0.12)$ & $2.1(2.32)$ & $19.1(19.3)$ & 375 (377) & $4.2(3.8)$ \\
\hline Ethanol & 795 (795) & $0.14(0.14)$ & $2.0(2.5)$ & $14.3(17.8)$ & 286 (349) & $4.3(3.44)$ \\
\hline Isobutanol & 797 & 0.14 & 2.08 & 14.9 & 275 & 4.1 \\
\hline DMSO & 791 & 0.17 & 2.86 & 16.8 & 220 & 2.9 \\
\hline Acetone & 788 (785.5) & $0.14(0.18)$ & $2.5(3.14)$ & $17.8(17.5)$ & $280(248)$ & $3.4(2.7)$ \\
\hline Acetophenone & 797 & 0.17 & 2.3 & 13.5 & 249 & 3.6 \\
\hline Dichloromethane & 788 & 0.08 & 2.34 & 29.75 & 280 & 3.9 \\
\hline Pyridine & 794 (793) & $0.20(0.26)$ & $2.9(3.53)$ & $14.5(13.8)$ & 193 (194) & $2.75(2.1)$ \\
\hline Quinoline & 803 & 0.15 & 2.4 & 16 & 237 & 3.54 \\
\hline Chloroform & 792 (796) & $0.09(0.17)$ & $1.7 \quad(2.6)$ & $18.9(15.3)$ & $244(258)$ & $5.3 \quad(3.2)$ \\
\hline Tetrahydrofuran & $782(782.5)$ & $0.20(0.24)$ & $3.0 \quad(3.56)$ & $15.0(14.8)$ & $166(190)$ & $2.66(2.1)$ \\
\hline Dioxan & 782 & 0.20 & 3.1 & 15.5 & 149 & 2.6 \\
\hline Diethyl ether & $781(782)$ & $0.14(0.19)$ & $2.66(3.0)$ & $19.0(16.0)$ & $183(183)$ & $3.2 \quad(2.5)$ \\
\hline
\end{tabular}

equation. The linear dependence of $v_{S t}=(500 \pm 180)$ $\mathrm{cm}^{-1} \cdot f(n, \epsilon)+$ const. was determined from the plot in Fig. 6 for BChl-ser (without considering the values in PBS and methanol because of their strong hydrogen bonding character) and BChl. It results in a dipole change $\Delta \mu=(2.5 \pm$ $0.3) \mathrm{D}$ for a minimal cavity radius of $a=5 \AA$. If a value of $a=8 \AA$ is used as an upper estimate, a dipole change of $\Delta \mu=5.1 \mathrm{D}$ is obtained $(25,26)$.

The fluorescence properties of BChl-ser are summarized in Table 2, together with those of BChl (in parenthesis, if available from the literature). Although most parameters are similar, there are some significant differences. Among these, the most obvious are a lower fluorescence yield and a shorter fluorescence lifetime for BChl-ser in most solvents. BChlser and BChl show the same radiative lifetime $\tau_{0}=\tau_{f l} \cdot \Phi_{f l}^{-1}$, taking into account an experimental error of $10 \%$. Apparently, the nonradiative rates (intersystem crossing - $k_{I S C}$, or internal conversion- $k_{I C}$ ) are enhanced in BChl-ser, in comparison with BChl.

In order to distinguish between both processes, fluorescence lifetimes were also measured at $77 \mathrm{~K}$ for $\mathrm{BChl}$ and BChl-ser in diethyl ether. An enhanced ISC quantum yield for BChl-ser $\left(\Phi_{I S C}=82 \%\right)$ compared with BChl $\left(\Phi_{I S C}=\right.$ $77 \%)$ was determined having $\Phi_{I S C}=1-\left(\tau_{f t}[77 \mathrm{~K}]\right) / \tau_{f t} \cdot \Phi_{f t}$ (27). The value for BChl is in good agreement with published data (7). The IC process is not influenced by the serine. In both molecules it is around $4 \%$, corresponding to an IC rate of about $0.15 \times 10^{8} \mathrm{~s}^{-1}$. In conclusion, the attached serine specifically enhances the ISC process. Excluding a radical pair mechanism, the enhancement probably originates from increased spin-orbit coupling, possibly by means of the bound water.

The fluorescence properties of BChl-ser dissolved in PBS can be summarized as follows: the Stokes shift is very large, with a value of $540 \mathrm{~cm}^{-1}$. The fluorescence quantum yield is small with $\Phi_{f l}=0.04$, and the fluorescence lifetime is comparably shortened to $\tau_{f}=1.1 \mathrm{~ns}$. This results in a radiative lifetime of $\tau_{0}=(27.5 \pm 3) \mathrm{ns}$, which can be determined by absorption data as well (28)

$$
\tau_{0}^{-1}=2.9 \cdot 10^{-9} n^{2}\left\langle v_{f}^{-3}\right\rangle^{-1} \int \frac{\epsilon(v)}{v} d \nu
$$

where $\left\langle v_{f}^{-3}\right\rangle^{-1}=\int I(v) \mathrm{d} v / \int v^{-3} I(v) \mathrm{d} \nu$ is the reciprocal of the mean value of $v^{-3}$ in the fluorescence spectrum. The radiative lifetime of $(30 \pm 3) \mathrm{ns}$ thus obtained for the $S_{1}$ state agrees within the error limits.

In an additional experiment, bacteriopheophorbide-serine (BPheide-ser) was dissolved in PBS in order to evaluate the contribution of the $\mathrm{Mg}$ atom on the solubility of BChl-ser in water. The absorption spectrum of BPheide-ser in PBS shows two absorption maxima at around 751 and $790 \mathrm{~nm}$ that are characteristic of a monomer-dimer equilibrium with the aggregate absorption redshifted with respect to the monomer (16). However, no fluorescence signal could be observed for BPheide-ser, which is also indicative of dimerization (29). Hence, the Mg atom plays a key role in enhancing the pigment solubility in water.

\section{CONCLUSIONS}

We have shown that the covalently bound amino acid, serine, influences the kinetics, but not the energetics, of the excited states of bacteriochlorophyll. The energy of the $S_{0}$ $\rightarrow S_{1}$ transitions and the Stokes shift of both BChl and BChlser show the same dependence on solvent polarities, but the deactivation processes from the $S_{1}$ state are somewhat enhanced in the serine conjugate. Moreover, a somewhat stronger ISC relaxation was found in BChl-ser by means of fluorescence quantum yield and fluorescence lifetime measurements, whereas the IC is comparable in both molecules.

The water environment, accessible with BChl-ser for the first time, leads to a larger Stokes shift, a reduced extinction coefficient at the maximum of the $Q_{y}(0,0)$ absorption band, a broadening of the absorption bands, a reduced fluorescence quantum yield and an increased nonradiative relaxation process when compared with nonaqueous solvents.

Acknowledgements - Support of this work by the German Israeli Foundation (project 1-497-140.1196) is gratefully acknowledged. This work has also been supported by the EU-TMR Program for the European Large Scale Laser Facilities Cluster (grant to A.S. for common research work at MBI).

\section{REFERENCES}

1. Henderson, B. W., A. B. Sumlin, B. L. Owczarczak and T. J. Dougherty (1991) Bacteriochlorophyll $a$ as photosensitizer for 
photodynamic treatment of transplantable murine tumors. $J$. Photochem. Photobiol. B: Biol. 10, 303-313.

2. Henderson, B. W., W. R. Potter, A. B. Sumlin, B. L. Owczarczak, F. S. Dougherty and T. J. Nowakowski (1990) Bacteriochlorophyll $a$ as photosensitizer for photodynamic treatment of transplantable murine tumors. SPIE, Photodyn. Ther.: Mech. II 1203, 211-222.

3. Rosenbach-Belkin, V., L. Chen, L. Fiedor, I. Tregub, F. Pavlotsky, V. Brumfeld, Y. Salomon and A. Scherz (1996) Serine conjugates of chlorophyll and bacteriochlorophyll: photocytotoxicity in vitro and tissue distribution in mice bearing melanoma tumors. Photochem. Photobiol. 64, 174-181.

4. Scherz, A., S. Katz, Y. Vakrat, V. Brumfeld, E. Gabelmann, D. Leupold, J. R. Norris, H. Scheer and Y. Salomon (1998) Bacteriochlorophyll-serine based photochemotherapy: type III PDT? In Photosynthesis: Mechanisms and Effects, Vol. V (Edited by G. Garab), pp. 4207-4212. Kluwer Academic, Dordrecht.

5. Fiedor L., V. Rosenbach-Belkin, M. Sai and A. Scherz (1996) Preparation of tetrapyrrole-amino acid covalent complexes. Plant Physiol. Biochem. 34, 393-398.

6. Limantara, L., S. Sakomoto, Y. Koyama and H. Nagae (1997) Effects of nonpolar and polar solvents on the $\mathrm{Q}_{x}$ and $\mathrm{Q}_{y}$ energies of bacteriochlorophyll $a$ and bacteriopheophytin $a$. Photochem. Photobiol. 65, 330-337.

7. Teuchner, K., H. Stiel, D. Leupold, I. Katheder and H. Scheer (1994) From chlorophyll- $a$ towards bacteriochlorophyll- $a$ : excited-state properties of modified pigments. J. Luminesc. 60/61, 520-522.

8. Teuchner, K., W. Becker and H. Stiel (1993) Multidecay fluorescence measurements with extended digital sampling device. Meas. Sci. Techn. Phys. 4, 1070.

9. Gouterman, M. and G. H. Wagnière (1963) Spectra of porphyrins, Part II. Four orbital model. J. Mol. Spectrosc. 11, 108127.

10. Hoff, A. J. and J. Amesz (1991) Visible absorption spectroscopy. In Chlorophylls (Edited by H. Scheer), pp. 723-738. CRC Press, Boca Raton.

11. Nagae, H., M. Kuki, R. J. Cogdell, Y. Koyama (1994) Shifts of the ${ }^{1} A_{g}^{-}$and ${ }^{1} B_{u}^{+}$electronic absorption state of carotenoids in nonpolar and polar solvents. J. Chem. Phys. 101, 6750-6765.

12. Lakowicz, J. R. (1983) Principles of Fluorescence Spectroscopy. Plenum Press, New York.

13. Hartwich, G., L. Fiedor, I. Simonin, E. Cmiel, W. Schaefer, D. Noy, A. Scherz and H. Scheer (1998) Metal-substituted bacteriochlorophylls. 1. Preparation and influence of metal and coordination on spectra. J. Am. Chem. Soc. 120, 3675-3683.

14. Koyama, Y. and L. Limantara (1998) Effects of singlet and triplet excitation, oxidation and axial coordination on the bond orders in the macrocycle of bacteriochlorophyll $a$ as revealed by resonance Raman spectroscopy. Spectrochim. Acta A 54, $1127-1139$.

15. Evans, T. A. and J. J. Katz (1975) Evidence for 5- and 6-coordinated magnesium in bacteriochlorophyll- $a$ from visible absorption spectroscopy. Biochim. Biophys. Acta 396, 414-426.

16. Scherz, A. and W. W. Parson (1984) Exciton interactions in dimers of bacteriochlorophyll and related molecules. Biochim. Biophys. Acta 766, 666-678.

17. Brereton, R. G. and K. M. Sanders (1983) Bacteriochlorophyll $a$ : influence of axial co-ordination on reactivity and stability. Design of an improved extraction procedure. J. Chem. Soc. Perkin Trans. I., 431-434.

18. Connolly, J. S., E. B. Samuel and A. F. Janzen (1982) Effects of solvent on the fluorescence properties of bacteriochlorophyll a. Photochem. Photobiol. 36, 565-574.

19. Katz, S. (1999) PhD thesis, The Weizmann Institute of Science.

20. Alden, R. G., E. Johnson, V. Nagarajan, W. W. Parson, C. J. Law and R. G. Cogdell (1997) Calculations of spectroscopic properties of the LH2 bacteriochlorophyll-protein antenna complex from Rhodopseudomonas acidophila. J. Phys. Chem. B 101, 4667-4680.

21. Reichardt, Ch. and K. Dimroth (1995) Lösungsmittel und empirische Parameter zur Charakterisierung ihrer Polarität Fortschr. chem. Forsch. 11(2), 1-73.

22. Kamlet, M. J., R. M. Doherty, R. W. Taft, M. H. Abraham and W. J. Koros (1984) Solubility properties in polymers and biological media. J. Am. Chem. Soc. 106, 1205-1212.

23. Abdel-Halim, S. T. (1993) Solvatochromisms of a typical merocyanine dye. J. Chem. Soc. Faraday Trans. 89, 55-57.

24. Kamlet, M. J., C. Dickinson and R. W. Taft (1981) Linear solvation energy relationships. Solvent effects on some fluorescence probes. Chem. Phys. Lett. 77, 69-72.

25. Liptay, W. (1965) Die Lösungsmittelabhngigkeit der Wellenzahl von Elektronenbanden und die chemisch-physikalischen Grundlagen. Z. Naturforsch. 20, 1441-1466.

26. Helenius, V. M., P. H. Hynninen and J. E. I. Korppi-Tommola (1993) Chlorophyll $a$ aggregates in hydrocarbon solution, a picosecond spectroscopy and molecular modeling study. Photochem. Photobiol. 58, 867-871.

27. Teuchner, K., A. Pfarrherr, H. Stiel, W. Freyer and D. Leupold (1993) Spectroscopic properties of potential sensitizers for new photodynamic therapy start mechanisms via two-step excited electronic states. Photochem. Photobiol. 57, 465-471.

28. Strickler, S. J. and R. A. Berg (1962) Relationship between absorption intensity and fluorescence lifetime of molecules. $J$. Chem. Phys. 37, 814-822.

29. Eichwurzel, I., H. Stiel and B. Roeder (2000) Photophysical studies of the pheophorbide $a$ dimer. J. Photochem. Photobiol. B: Biol. 54(2/3), 194-200.

30. Lavery, H., R. Sklenar, K. Zakrzewska and B. Pullman (1986) The flexibility of the nucleic acids: (II). The calculation of internal energy and applications to mononucleotide repeat DNA. J. Biomol. Dyn. 3, 989-1014. 